\title{
Editorial
}

\section{Themenschwerpunkt Leitlinien für Diagnostik und Therapie in der Neuropsychologie}

\author{
Sandra Verena Müller ${ }^{1}$ und Angelika Thöne-Otto² (Leitlinienbeauftragte der GNP) \\ Fakultät Soziale Arbeit, Ostfalia Hochschule für angewandte Wissenschaften, Wolfenbüttel \\ Tagesklinik für Kognitive Neurologie, Universitätsklinikum Leipzig
}

Liebe Leserinnen und Leser,

Sie mögen sich vielleicht fragen: Brauchen wir in der Zeitschrift für Neuropsychologie ein Themenheft Leitlinien? Oder: Wozu brauchen wir in der Neuropsychologie Leitlinien? Oder vielleicht auch: Engen die Leitlinien möglicherweise meine diagnostische oder therapeutische Freiheit ein? Schon einmal vorab: das sicher nicht, denn Leitlinien sind Entscheidungshilfen, keine Richtlinien. Leitlinien dienen vor allem der Vermittlung von Wissen: Transfer von Evidenz über Empfehlungen in die Praxis. Weiterhin besteht über die Anforderungen an die Qualität von Leitlinien international Konsens. Sie dienen der bestmöglichen Versorgung der Patientinnen und Patienten, also der Qualitätssicherung.

Nun gehen wir noch mal einen Schritt zurück in die neuropsychologische Leitlinienentwicklung im deutschsprachigen Raum. Dabei wird deutlich, wie schnell die Zeit vergeht! Nachdem 2005 die „Leitlinien der Gesellschaft für Neuropsychologie (GNP) für neuropsychologische Diagnostik und Therapie“ in der Zeitschrift für Neuropsychologie erschienen waren, wurden die neuropsychologischen Themen Aufmerksamkeit, Gedächtnis, Exekutivfunktionen und Raumkognition 2008 erstmals unter Federführung von Neuropsychologinnen und Neuropsychologen in die vierte Auflage des Buches „Leitlinien für Diagnostik und Therapie in der Neurologie“, herausgegeben von Hans-Christoph Diener und Norman Putzki im Auftrag der Deutschen Gesellschaft für Neurologie (DGN), aufgenommen. Claus-Werner Wallesch hat als Mitglied der DGN uns als GNP damals diese Türe geöffnet, die Federführung aber bei den Neuropsychologen gelassen. Für diese Unterstützung sei ihm herzlich gedankt. Diese Leitlinien erreichten das Evidenzniveau S1 als Expertenempfehlungen, deren Konsensfindung nach einem informellen Verfahren erfolgt. Damals gab es die Leilinien ausschließlich in gebundener Form, also als dickes und teures Buch.

Als im Jahr 2012 die fünfte, vollständig überarbeitete Auflage der ,Leitlinien für Diagnostik und Therapie in der Neurologie" erschien, wurden sie schon parallel in Buchform und als Download auf den Internetseiten der DGN und der Arbeitsgemeinschaft der Wissenschaftlichen Medizinischen Fachgesellschaften e.V. (AWMF) veröffentlicht. Drei der vier „neuropsychologischen“ Leitlinien erreichten zu diesem Zeitpunkt das Evidenzniveau S2 evidenzbasiert, kurz S2e. Eine S2e-Leitlinie ist eine evidenzbasierte Leitlinie, deren Entwicklung auf systematischer Recherche, Auswahl und Bewertung der Literatur erfolgt. Seither ist eine Reihe von Leitlinien erschienen, bei denen Neuropsychologinnen und Neuropsychologen vom GNP-Vorstand in die jeweiligen Redaktionskomitees entsandt wurden. Oft leisten die Kolleginnen und Kollegen im Konsensusprozess wertvolle und nachhaltige Arbeit, damit neuropsychologische Ansätze in den Empfehlungen angemessen vorkommen. Einen Konsens der verschiedenen Interessenvertretenden zu finden, zeichnet den Leitlinienfindungsprozess aus, und es ist gleichzeitig oft eine Herausforderung, bei der um einzelne Begriffe und Formulierungen gerungen wird. Ihnen allen sei an dieser Stelle herzlich gedankt.

Seit den ersten Diskussionen um die Angemessenheit evidenzbasierter Ansätze für die Neuropsychologie (z.B. Dick \& Kringler, 2007; Hildebrandt, Müller \& Schwendemann, 2004) hat sich viel getan, insbesondere die Methodik der durchgeführten Therapiestudien hat sich enorm verbessert. Daher sind wir zuversichtlich, bei der nächsten Auflage das höchste Niveau der S3-Leitlinien zu erreichen. Über die Jahre hat sich nicht nur die Sichtbarkeit der 
Leitlinien erhöht, sondern auch deren Verfügbarkeit verbessert. Dennoch halten wir ein Sonderheft zum Thema Leitlinien für sinnvoll, um die wichtigsten Empfehlungen für Sie unmittelbar verfügbar zu machen. Das Regelwerk zur Leitlinienerstellung wurde in den Jahren immer komplexer, was in Folge die Erstellung und den Konsensusprozess aufwendiger macht. Umso mehr freuen wir uns, Ihnen heute endlich Kurzversionen der gänzlich neu überarbeiteten „neuropsychologischen“ Leitlinien präsentieren zu dürfen. Sie finden in diesem Heft Kurzfassungen zu den Themenkomplexen Raumkognition (federführende Autoren: Hans-Otto Karnath und Josef Zihl), Gedächtnis (federführende Autorin: Angelika ThöneOtto) und Exekutivfunktionen (federführende Autorin und Autor: Sandra Müller und Tilmann Klein) sowie zu der für die Neuropsychologie wichtigen Leitlinie „Begutachtung bei gedecktem Schädel-Hirntrauma (federführender Autor: Claus-Werner Wallesch). Das Thema Aufmerksamkeit befindet sich noch in Bearbeitung. Leider können wir hier nicht alle Leitlinien, bei denen wir als Fachgesellschaft beteiligt waren, vorstellen. Sie finden diese auf der Homepage der GNP (https://www.gnp.de/fachinformati onen/leitlinien).

Unser Anliegen ist es, dass die wissenschaftlichen Erkenntnisse, die die Basis für die Leitlinienempfehlungen darstellen, zunehmend Einzug in die neuropsychologische Diagnostik und Therapie halten. Sie sollen uns eine wis- senschaftliche Grundlage geben in der Verhandlung von Struktur- und Prozessqualitätsmerkmalen, beitragen zur weiteren Verbesserung der klinischen Versorgung und letztlich zum Wohle der behandelten Menschen.

im Juni 2020

Sandra Verena Müller \& Angelika Thöne-Otto (Leitlinienbeauftragte der GNP)

\section{Literatur}

Dick, F. \& Kringler, W. (2007). Evidenzbasierung, Methodik, therapeutische Freiheit und Kreativität. Zeitschrift für Neuropsychologie, 18, 41-54.

Hildebrandt, H., Müller, S.V. \& Schwendemann, G. (2004). Evidenzbasierte neuropsychologische Therapie. Neurologie und Rehabilitation, 10, 57-68.

Prof. Dr. rer. nat. habil. Sandra Verena Müller

Rehabilitation und Teilhabe

Fakultät Soziale Arbeit

Ostfalia Hochschule für angewandte Wissenschaften

Salzdahlumer Str. 46/48

38302 Wolfenbüttel

Deutschland

s-v.mueller@ostfalia.de 